\title{
Efeito da umidade da superfície do solo no desempenho do método de Penman original e modificado ${ }^{1}$
}

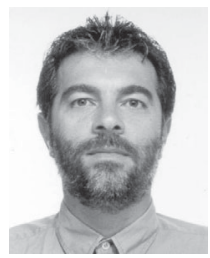

\author{
Dalva M. Curi Lunardi ${ }^{2}$, Marco A. Lunardi ${ }^{3}$ \& Marco A. Furlan ${ }^{4}$
}



Protocolo $083-6 / 6 / 2001$

\begin{abstract}
Resumo: Neste estudo, avaliou-se o efeito da umidade da superfície do solo na precisão de estimativa da evapotranspiração de referência ET o pelo método de Penman original e modificado, através de duas funções de vento, sendo a evapotranspiração medida com lisímetros de nível freático. No Período I, a área externa aos lisímetros não recebeu irrigação e a superfície dos lisímetros permaneceu seca, estando o nível freático a $46 \mathrm{~cm}$ de profundidade. Nos dias com precipitação, os mesmos foram cobertos com plástico. No Período II, a área de bordadura recebeu irrigação e a profundidade do nível freático, $30 \mathrm{~cm}$, permitiu que a sua superfície permanecesse úmida. No Período I, o método de Penman superestimou a evapotranspiração e a função de vento gerada apresentou-se fisicamente inconsistente; no Período II, tanto o método original quanto o modificado revelaram alta precisão, demonstrando a necessidade de se manter a umidade da superfície do solo constante.
\end{abstract}

Palavras-chave: água disponível, lisímetros, evapotranspiração

\section{Effect of soil surface moisture content on the performance of original and modified Penman method}

\begin{abstract}
This study evaluated the effect of soil surface moisture on the performance of the original and modified Penman method by two-wind function. The evapotranspiration was obtained with constant water table lysimeters. In Period I, the border area of lysimeter was wetted by precipitation only and due to water table depth $(46 \mathrm{~cm})$ the soil surface remained dry. A plastic cover was placed on the lysimeters during the rainy days. During the Period II, the border area of lysimeter was irrigated and water table depth at $30 \mathrm{~cm}$ allowed soil surface to be maintained wet. In Period I, the Penman method overestimated evapotranspiration and the obtained wind function showed to be physically inconsistent. In Period II, the original and modified Penman methods showed better performance demonstrating the importance of maintaining moisture content of soil surface constant.
\end{abstract}

Key words: available water, Iysimeters, evapotranspiration

\section{INTRODUÇÃO}

Diversos são os métodos para a estimativa da evapotranspiração de referência (ETo), sendo a escolha função da disponibilidade de dados climáticos, da precisão necessária, da conveniência e do custo. Pereira et al. (1997) agruparam esses métodos em cinco categorias: empíricos, aerodinâmicos, do balanço de energia, combinados e da correlação dos turbilhões.

Dentre os combinados, o método original de Penman (1948) é um dos mais empregados, cuja fórmula foi derivada assumindo proporcionalidade entre a evaporação da água e a evapotranspiração da grama sub-irrigada. Usando a relação entre os fluxos de calor sensível e latente, descrita por Bowen em 1926, e o poder evaporante do ar à sombra, Penman, eliminou medidas de superfície, inexistentes em estações meteorológicas convencionais.
A sua tendência de superestimativas em várias partes do mundo (Smith, 1991) também foi constatada no Planalto Paulista (Camargo \& Sentelhas, 1997). Sedyama (1996) e Sentelhas (1998) atribuíram, como fatores responsáveis pela inadequação da função do vento para as condições locais, o método de cálculo do déficit de pressão de vapor e a ausência de resistência exercida pela cobertura vegetal, enquanto Tanner \& Pelton (1960) apontaram, como causas da sobre-estimativa, o tamanho insuficiente da bordadura, ausência de medições da temperatura acima da cultura, armazenamento de calor sensível e déficit de umidade no solo. Penman, citado pelos mesmos autores, considerou que a determinação do saldo de radiação $(\mathrm{Rn})$ é mais importante, principalmente quando a radiação incidente $(\mathrm{Rg})$ é medida e a emissão de ondas longas (Rl) é estimada. Quando ambas são estimadas, devido ao erro relativo ser na 
mesma ordem de magnitude em nível diário, a precisão na determinação de Rn é maior.

Pereira et al. (1996) avaliaram os erros na determinação do poder evaporante do ar à sombra (Ea), dado pelo produto de uma função empírica da velocidade do vento - $\mathrm{f}(\mathrm{U})$ representada por uma regressão linear e pelo déficit de pressão de vapor $(\Delta \mathrm{e})$. Dentre os motivos, os autores atribuíram o fato da função do vento ter sido originada, por Penman (1948), acima de um tanque de evaporação, embora tanto a velocidade do vento quanto o déficit de pressão de vapor fossem medidos acima da vegetação.

Após revisarem muitos trabalhos, Doorenbos \& Pruitt (1977) propuseram uma nova função de vento, tendo a grama como cultura de referência. Para eles, a função proposta por Penman depende da maneira de determinação do déficit de saturação médio diário $(\Delta \mathrm{e})$.

Maniero (1993) testou seis métodos de estimativa do déficit de pressão de vapor $(\Delta \mathrm{e})$, observando que todos apresentaram boa correlação com o método padrão, no qual, o déficit de pressão de vapor médio diário é calculado usando-se a média horária de $\Delta \mathrm{e}$ no período entre às $7 \mathrm{e} 18 \mathrm{~h}$. Desta forma, recomendou utilizar o método mais simples, cujas observações foram feitas às $9 \mathrm{e} 15 \mathrm{~h}$.

Pereira et al. (1996) utilizando um lisímetro de nível freático constante cultivado com grama, observaram que, independente do método de estimativa de $\Delta \mathrm{e}$, a função original de Penman superestimou, em torno de $20 \%$, os valores de ETo. Neste trabalho para cada um dos seis métodos de estimativa de $\Delta \mathrm{e}$ empregados, foram obtidas duas funções de vento, com coeficientes lineares variando de 0,41 a 1,0 no verão e de $-0,07$ a 0,12 na primavera e outono. Os autores não atribuíram esta variação à altura da grama, justificando que a aerodinâmica da superfície afeta somente o coeficiente angular.

Este trabalho teve como objetivo avaliar o efeito da umidade da superfície do solo na precisão do método Penman, conforme proposto em 1948 e modificado pelo emprego de duas funções de vento obtidas. Estas estimativas foram comparadas com medidas da ETo obtidas com lisímetros de nível freático constante.

\section{MATERIAL E MÉTODOS}

O trabalho de campo foi conduzido na área experimental do Departamento de Recursos Naturais da Faculdade de Ciências Agronômicas, UNESP, no município de Botucatu, SP, com as seguintes coordenadas geográficas: $22^{\circ} 51^{\prime} \mathrm{S}$ de latitude, $48^{\circ} 26^{\prime} \mathrm{W}$ de longitude e $786 \mathrm{~m}$ de altitude.

O clima local, segundo classificação de Köeppen, é Cwa: clima quente (mesotérmico) com chuvas no verão e estação seca no inverno, sendo a temperatura do mês mais quente superior a $22^{\circ} \mathrm{C}$.

Os dados climáticos utilizados foram provenientes de uma estação meteorológica situada próxima à estação lisimétrica e o índice de área foliar foi determinado com um medidor modelo Ci202 (U.S.A) retirando-se uma área de $100 \mathrm{~cm}^{2}$ de grama de cada lisímetro, nos diferentes períodos analisados.

A evapotranspiração foi medida numa estação lisimétrica com 5 conjuntos lisimétricos de nível freático, instalados no centro de uma área de $6.700 \mathrm{~m}^{2}$, cultivados com grama (Paspalum notatum F.). Cada conjunto era composto por uma caixa de cimento amianto com $2 \mathrm{~m}^{2}$ de área interna, um tanque intermediário e um tanque medidor (Cury-Lunardi, 2000).
No Período I, janeiro de 1996 a outubro de 1998, a fim de que a superfície do solo permanecesse seca, o nível freático nos lisímetros foi mantido a $46 \mathrm{~cm}$ de profundidade. A disponibilidade hídrica a $20 \mathrm{~cm}$ foi mantida constante pelo fornecimento de água pelo nível freático. Nos dias com precipitação, a superfície evapotranspirante era coberta e os dados excluídos nos cálculos. A área externa aos lisímetros só recebeu água por precipitação.

No Período II, de novembro de 1998 a novembro de 1999, a área externa foi irrigada com freqüência determinada através das medidas lisimétricas e da tensão de água no solo, dentro e fora dos lisímetros. O nível freático foi mantido a $30 \mathrm{~cm}$, possibilitando permanente umidade na superfície do solo.

Os dois períodos foram subdivididos em estação seca, de abril a setembro, e chuvosa, de outubro a março. A evapotranspiração de referência foi estimada pelo método de Penman (1948), utilizando-se a seguinte equação:

$$
\mathrm{ET}_{0}=\frac{\mathrm{WRn}}{\lambda}+(1-\mathrm{W}) \mathrm{Ea}
$$

sendo: $\mathrm{ET}_{\mathrm{o}}$, evapotranspiração em $\mathrm{mm} \mathrm{d}^{-1}, \lambda$ calor latente de evaporação $\mathrm{MJ} \mathrm{kg}^{-1}, \mathrm{Rn}$ (saldo de radiação) em $\mathrm{MJ} \mathrm{m}^{-2} \mathrm{~d}^{-1}$, $\mathrm{W}$ fator de ponderação dependente da temperatura do ar e Ea o poder evaporante do ar em $\mathrm{MJ} \mathrm{m}^{2} \mathrm{~d}^{-1}$ dado por:

$$
\mathrm{Ea}=\mathrm{f}(\mathrm{U}) \Delta \mathrm{e}
$$

sendo: $\Delta$ e o déficit de pressão de vapor em $\mathrm{kPa}$ e $\mathrm{f}(\mathrm{U})$ dado pelo modelo:

$$
f(U)=m(a+b U)
$$

que, segundo Penman (1948), assume os seguintes valores:

$$
f(U)=6,43(1+0,526 U)
$$

donde: $\mathrm{U}$ é a velocidade do vento a 2,0 $\mathrm{m}_{\text {de }}$ altura $\mathrm{em} \mathrm{m} \mathrm{s}^{-1}, \mathrm{~m}$ em MJ m ${ }^{-2} \mathrm{~d}^{-1} \mathrm{kPa}^{-1}$, a é adimensional e $\mathrm{b}$ em $\mathrm{s} \mathrm{m}^{-1}$.

A função da velocidade do vento foi gerada para os dois períodos isolando-se $\mathrm{f}(\mathrm{U})$ na Eq. (1) como se segue:

$$
\mathrm{f}(\mathrm{U})=\frac{\mathrm{ET}_{\mathrm{o}}-\frac{\mathrm{WRn}}{\lambda}}{(1-\mathrm{W}) \Delta \mathrm{e}}
$$

sendo: $\mathrm{ET}_{\mathrm{o}} \mathrm{o}$ valor diário da evapotranspiração medida.

O déficit de pressão de vapor foi obtido da seguinte forma:

$$
\Delta \mathrm{e}=\mathrm{es}(\mathrm{T})-(\mathrm{U} \operatorname{Res}(\mathrm{T}))
$$

sendo: UR, umidade relativa média diária (\%) e T a temperatura média diária $\left({ }^{\circ} \mathrm{C}\right)$, ambos obtidos através da média de 24 leituras a cada hora do dia. 
Os valores diários de $\mathrm{f}(\mathrm{U})$ foram correlacionados com a velocidade do vento $\mathrm{U}$, em $\mathrm{m} \mathrm{s}^{-1}$, através de regressão linear simples. Para comparação dos coeficientes da equação com os recomendados por Penman (Eq. 4), necessitou-se estabelecer o coeficiente $\mathrm{m}$. Para isto, adotou-se $\mathrm{m}=\mathrm{a}$, dividindo-se $\mathrm{b}$ por $\mathrm{a}$, sendo, conseqüentemente, $\mathrm{a}=1$.

Para gerar as equações de $\mathrm{f}(\mathrm{U})$ do Período I foram utilizados 234 d e, do Período II, 138 d.

Para serem validadas com dados independentes do mesmo período, as duas equações foram incorporadas ao método de Penman, estimando-se a ETo em 730 d no Período I e 177 d no Período II.

Os resultados obtidos foram avaliados através de análise de regressão entre os valores medidos e estimados da ETo, por meio dos coeficientes angular b e de determinação $\mathrm{R}^{2}$.

\section{RESULTADOS E DISCUSSÃO}

Nos dois períodos analisados, a grama dentro dos lisímetros teve bom desenvolvimento, devido à presença do nível freático, mostrado através do índice de área foliar na Tabela 1.

Tabela 1. Valores do índice de área foliar médio da grama, com $0,12 \mathrm{~m}$ de altura, para as estações seca e chuvosa dos dois períodos

\begin{tabular}{ccc}
\hline \multirow{2}{*}{ Período } & \multicolumn{2}{c}{ Estação } \\
\cline { 2 - 3 } & Seca & Chuvosa \\
\hline I & 6,0 & 6,9 \\
II & 6,1 & 6,8 \\
\hline
\end{tabular}

Embora a grama se tenha desenvolvido bem nos dois períodos, observa-se, através da Tabela 2, que a evapotranspiração medida no Período I foi restrita devido à interrupção da capilaridade nos primeiros centímetros de solo, com diminuição da evaporação, ao contrário do ocorrido no Período II.

Tabela 2. Valores médios de evapotranspiração medida $\left(\mathrm{mm} \mathrm{d}^{-1}\right)$, nos dois períodos

\begin{tabular}{cccc}
\hline \multirow{2}{*}{ Período } & \multirow{2}{*}{ Período Total } & \multicolumn{2}{c}{ Estação } \\
\cline { 3 - 4 } & & Seca & Chuvosa \\
\hline I & 2,2 & 2,7 & 1,6 \\
II & 3,0 & 3,8 & 2,3 \\
\hline
\end{tabular}

\section{Determinação da função empírica do vento f(U)}

Sendo o método de Penman composto de um termo energético, que expressa o efeito da radiação solar sobre a evapotranspiração, e outro, que representa as condições aerodinâmicas da superfície combinando os processos físicos de balanço de energia com processos turbulentos de transferência de massa, a função do vento depende, fundamentalmente, das características aerodinâmicas da superfície (Smith 1991).

Como a equação de $\mathrm{f}(\mathrm{U})$ proposta por Penman representa um coeficiente de transporte global de vapor d'água na atmosfera, podendo não se aplicar com eficiência em todas as condições, sendo apontada por Pereira et al. (1997) como uma das causas de sobreestimativa do método, procurou-se analisar as diferenças nos modelos de $\mathrm{f}(\mathrm{U})$ gerados nos dois períodos.
Embora existam diversas maneiras de se calcular o déficit de pressão de vapor $(\Delta \mathrm{e})$ e que, evidentemente, influenciam nos valores dos coeficientes das equações, optou-se por empregar apenas uma, já que se pretendia apenas comparar o desempenho do método, com o seu emprego, nos dois períodos.

A tentativa de se determinar uma função para cada estação do ano, não se mostrou precisa, ficando estabelecida uma única função para cada período analisado.

Para efeito de comparação com as equações de uso consagrado, ou seja, Penman (1948) e Doorenbos \& Pruitt (1977), o coeficiente a foi considerado igual a 1, para se obter o valor do coeficiente $\mathrm{m}$, determinando-se as seguintes equações para os períodos I e II, respectivamente:

$$
f(U)=-3,96(1-0,135 U)
$$

$$
f(U)=4,12(1+0,614 U)
$$

sendo $\mathrm{m}$ dado em $\mathrm{MJ} \mathrm{m}^{-2} \mathrm{~d}^{-1} \mathrm{kPa}^{-1}$, b em s m${ }^{-1}$ e $\mathrm{U}_{\text {em m s}}{ }^{-1}$.

Observa-se que os coeficientes das duas equações foram diferentes e que no Período I, Eq. 7, o coeficiente m assumiu valor negativo. Como para a obtenção de $m$ o coeficiente $b$ foi dividido por a, este também passou a apresentar valores negativos, embora não signifique inclinação negativa da reta.

Pereira et al. (1996) também obtiveram valores negativos do coeficiente $m$ da equação de $\mathrm{f}(\mathrm{U})$ no outono, em Araras, SP, atribuindo-os aos baixos valores da ETo. Maniero (1993) apontou como causa o repouso do gramado, embora não tenha citado os valores do IAF ao longo das estações. No presente trabalho, os baixos valores de evapotranspiração foram devidos à forma de obtenção de $\mathrm{f}(\mathrm{U})$ e à pequena evaporação do solo, que é função da profundidade do nível freático adotada. Não é comum considerar-se que o gramado estivesse em repouso, tendo em vista que o IAF foi bem acima daqueles citados por Jensen et al. (1990), de 1,9 a 3,6 e por Smith (1991) igual a 2.88.

No Período II, o menor valor do coeficiente $\mathrm{m}$, comparadose com o de Penman (1948) que é igual a 6,43 $\mathrm{MJ} \mathrm{m}^{-2} \mathrm{~d}^{-1} \mathrm{kPa}^{-1} \mathrm{e}$ Doorenbos \& Pruitt (1977), 6,61 MJ m ${ }^{-2} \mathrm{~d}^{-1} \mathrm{kPa}^{-1}$, pode ser atribuído à forma de obtenção de $\Delta \mathrm{e}$, às diferenças climáticas entre os locais e à época de estudo. Hargreaves (1983), ao proceder esta mesma comparação, observou que, nesses trabalhos, os autores não especificaram a época do ano em que os mesmos foram desenvolvidos nem o tipo de grama empregada.

Choisnel et al. (1992) e Steiner et al. (1991) consideraram que a resistência da cultura ao transporte de vapor d'água pode variar consideravelmente entre os cortes da grama, sendo dependente da espécie.

Sendo a inclinação da reta função da aerodinâmica da superfície, justifica-se, com isto, ter-se obtido maior valor de b que os recomendados por Penman (1948) já que as espécies de grama são distintas.

O aumento de b no Período II em relação ao Período I, pode ser justificado pela diminuição da resistência à difusão de vapor d'água, em razão da diminuição da temperatura da superfície, devido a mesma estar constantemente úmida. 


\section{Desempenho do método de Penman}

A equação de Penman (1948) foi desenvolvida para se estimar a evapotranspiração de uma superfície úmida em desenvolvimento ativo, com bordadura suficiente a evitar energia advectiva e sem deficiência hídrica.

Como o autor admitiu que a pressão saturante de vapor da superfície poderia ser obtida pela temperatura do ar, com ausência de umidade na superfície do solo, como no Período I, a temperatura da superfície é subestimada por se adotar, nos cálculos, a temperatura do ar, sendo a pressão de vapor acima desta não saturante, caso em que ocorre subestimativa de calor sensível transferido para atmosfera com sobreestimativa do método original, conforme Tabela 3.

Tabela 3. Coeficientes de determinação $\left(\mathrm{r}^{2}\right)$ e angular (b) da regressão linear entre os valores de ETo medidos com os lisímetros e os estimados pelo método original de Penman e com a Eq. (7) de estimativa de f(U), para o Período I

\begin{tabular}{ccccc}
\hline \multirow{2}{*}{ Período I } & \multicolumn{4}{c}{ Método } \\
\cline { 2 - 5 } & \multicolumn{3}{c}{ Original } & \multicolumn{2}{c}{$\mathrm{f}(\mathrm{U})$ Eq. (7) } \\
\cline { 2 - 5 } Total & $\mathrm{r}^{2}$ & $\mathrm{~b}$ & $\mathrm{r}^{2}$ & $\mathrm{~b}$ \\
\hline Est. seca & 0,96 & 1,69 & 0,96 & 1,11 \\
Est. chuvosa & 0,97 & 1,93 & 0,94 & 1,16 \\
& 0,97 & 1,59 & 0,97 & 1,09 \\
\hline
\end{tabular}

Substituindo-se, no método, a equação de $\mathrm{f}(\mathrm{U})$ proposta por Penman pela obtida para o Período I, observa-se que, embora fisicamente inconsistente e devido aos valores negativos de seus coeficientes, a mesma sobreestimou apenas $11 \%$, considerando o período como um todo, conforme Tabela 3.

Embora alguns autores concordem quanto à não universalidade da equação original de $f(U)$ por apresentar coeficiente de transporte sem especificar claramente as características aerodinâmicas da superfície, não se poderia esperar que, no Período I, o seu desempenho fosse satisfatório, já que as condições experimentais não satisfaziam os pré-requisitos para se obter a evapotranspiração de referência. A restrição da evaporação do solo com supremacia da transpiração, processo este dependente da conjunção de uma série de fatores biológicos e ambientais e o bom desempenho da função de vento obtida, embora fisicamente inconsistente, demonstram que, nestas condições, outro modelo matemático e físico, que não o de Penman, deveria ser empregado na estimativa da evapotranspiração. Desta forma, deve-se questionar a validade de trabalhos que discutem a imprecisão do método de Penman, ou propõem funções de vento quando a energia advectiva contribui no processo de perda d'água e os valores medidos não são devidos à evaporação do solo mas à transpiração da planta. Esses problemas são comuns quando se empregam lisímetros de nível freático constante e não se mantém a superfície do solo úmida.

No Período II observa-se, através da Tabela 4, que o desempenho do método original foi excelente em todas as estações.

Com o emprego da equação de $f(U)$ proposta, houve menor exatidão revelada pelo coeficiente $b$ e aumento de precisão. Desta forma, os resultados confirmam as avaliações favoráveis feitas ao método original de Penman em diversas partes do mundo, desde que as condições de manejo sejam adequadas.
Tabela 4. Coeficientes de determinação $\left(\mathrm{r}^{2}\right)$ e angular (b) da regressão linear entre os valores medidos com lisímetro e os estimados, pelo método original de Penman e com a Eq. (8) de estimativa de $\mathrm{f}(\mathrm{U})$ para o Período II

\begin{tabular}{ccccc}
\hline & \multicolumn{5}{c}{ Método } \\
\cline { 2 - 5 } Período II & \multicolumn{3}{c}{ Original } & \multicolumn{2}{c}{$\mathrm{f}(\mathrm{U})$} & $\mathrm{Eq} .(8)$ \\
\cline { 2 - 5 } & $\mathrm{r}^{2}$ & $\mathrm{~b}$ & $\mathrm{r}^{2}$ & $\mathrm{~b}$ \\
\hline Total & 0,93 & 0,96 & 0,96 & 0,87 \\
Estação seca & 0,99 & 1,02 & 0,99 & 0,92 \\
Est. Chuvosa & 0,90 & 0,93 & 0,94 & 0,85 \\
\hline
\end{tabular}

\section{CONCLUSÕES}

1. Embora as características construtivas do lisímetro de nível freático constante permitam que a irrigação superficial seja dispensada, nestas condições, a pouca evaporação da água do solo ou a lentidão na ascensão capilar, por interrupção da capilaridade, provocou o prevalecimento da transpiração, ocasionando propostas errôneas de ajustes empíricos, obtidos como resíduo da aplicação dos modelos tradicionais, como o de Penman e valores medidos com esses equipamentos.

2. Durante a estação seca, de abril a setembro e desde que as condições hídricas sejam satisfeitas, o desenvolvimento da grama para a região em estudo permite a determinação da evapotranspiração de referência.

3. Para que o método original de Penman tenha desempenho satisfatório, a manutenção permanente da umidade adequada da superfície é um dos fatores fundamentais.

4. Numa mesma área experimental, o teor de umidade da superfície do solo determina diferentes modelos de ajustes dos parâmetros empíricos, como $\mathrm{f}(\mathrm{U})$ da equação original de Penman.

5. Com a equação de $f(U)$ proposta no Período II, obteve-se maior precisão sem, contudo, ter ocorrido aumento de exatidão do método de Penman.

\section{AGRADECIMENTOS}

O segundo autor agradece a FAPESP, através do Processo $\mathrm{N}^{\circ} 98 / 15085-0$.

\section{LITERATURA CITADA}

Camargo, A.P.; Sentelhas, P.C. Avaliação do desempenho de diferentes métodos de estimativa da evapotranspiração potencial no Estado de São Paulo. Revista Brasileira de Agrometeorologia, Santa Maria, v.5, n.1, p.89-97, 1997.

Choisnel, E.; de Villele, O.; Lacrose, F. Une Approche uniformise è du calculo de l'évapotranspiration potentielle por 1'ensemble des pays de la communautè Europeénne, Communauté Commun Européennes, EUR 144233 Fr, Luxembourg, 1992.176p.

Cury-Lunardi, D.M. Efeito da condição de umidade da superfície do solo na evapotranspiração de referência medida e estimada. Botucatu: UNESP, 2000. 103p. Tese Livre Docência

Doorenbos, J.; Pruitt, W.O. Guidelines for predicting crop water requirements. Rome: FAO, 1977. 179p. Irrigation and Drainage, Paper 24 
Hargreaves,G.H. Discussion of application of Penman wind function by H. Cuenca and M.T. Nicholson. Journal Irrigation and Drainage Division Asce. New York, v.102, n.2, p.277-278, 1983.

Jensen, M.E.; Bruman, R.D.; Allen, R. G. Evapotranspiration and irrigation water requirements. New York: ASCE, 332p. Manual and reports 70

Maniero, M.A. Análise da função velocidade do vento na equação de Penman. Piracicaba, ESALQ, 1993. 69p. Dissertação Mestrado

Penman, H.L. Natural evaporation from open water, bare soil and grass. Proceedings of the Royal Society of London, v.A193, p.120-146, 1948.

Pereira, A.R.; Maniero, M.A.; Villa Nova, N.A.; Barbieri, V. Penman's wind function for a tropical humid climate. Revista Brasileira de Agrometeorologia, Santa Maria, v.4, n.1, p.69-75, 1996.
Pereira, A.R.; Villa Nova, N.A.; Sediyama, G.C. Evapotranspiração. Piracicaba: FEALQ, 1997. 183p.

Sediyama, G.C. Estimativa de evapotranspiração: histórico, evolução e análise crítica. Revista Brasileira de Agrometeorologia, Santa Maria, v.4,n.1,p.1-14, 1996.

Sentelhas, P.S. Estimativa da evapotranspiração de referência com dados de estação meteorológica convencional e automática. Piracicaba: ESALQ, 1998. 97p. Tese Doutorado

Smith, M. Report on expert consultation on procedures for revision of FAO methodologies for crop water requirements. Rome: FAO, 1991.45p.

Steiner, J.L.; Howel, T.A.; Schneider, A.D. Lysimetric evaluation of daily potential evapotranspiration models for grain sorghum. Agronomy Journal, Madison, v.83, p.240-7, 1991.

Tanner, C.B.; Pelton, W.L. Potential evapotranspiration estimates by the approximate energy balance method of Penman. Journal of Geophysical Research, Ottawa, v.65, n.10, p.391-413, 1960. 